\title{
BMI Trajectories as a Harbinger of Pre-Diabetes or Underdiagnosed Diabetes: an 18-Year Retrospective Cohort Study in Taiwan
}

\author{
Ching-Ju Chiu, $P h D^{7}$, Siao-Ling Li, MS ${ }^{7}$, Chih-Hsing $W u, M D^{1,2}$, and Ye-Fong Du, MD ${ }^{3}$ \\ ${ }^{1}$ Institute of Gerontology, College of Medicine, National Cheng Kung University, Tainan, Taiwan; ${ }^{2}$ Department of Family Medicine, National Cheng \\ Kung University Hospital, Tainan, Taiwan; ${ }^{3}$ Division of Endocrinology and Metabolism, Department of Internal Medicine, National Cheng Kung \\ University Hospital, Tainan, Taiwan.
}

\begin{abstract}
BACKGROUND: Although prior studies have examined BMI trajectories in Western populations, little is known regarding how BMI trajectories in Asian populations vary between adults with and without diabetes.

OBJECTIVE: To examine how BMI trajectories vary between those developing and not developing diabetes over 18 years in an Asian cohort.

DESIGN: Multilevel modeling was used to depict levels and rates of change in BMI for up to 18 years for participants with and without self-reported physiciandiagnosed diabetes.
\end{abstract}

PARTICIPANTS: We used 14,490 data points available from repeated measurements of 3776 participants aged $50+$ at baseline without diabetes from a nationally representative survey of the Taiwan Longitudinal Study on Aging (TLSA1989-2007).

MAIN MEASURES: We defined development of diabetes as participants who first reported diabetes diagnoses in 2007 but had no diabetes diagnoses at baseline. We defined the reference group as those participants who reported the absence of diabetes at baseline and during the entire follow-up period.

KEY RESULTS: When adjusted for time-varying comorbidities and behavioral factors, higher level and constant increases in BMI were present more than 6.5 years before self-reported diabetes diagnosis. The higher BMI level associating with the development of diabetes was especially evident in females. Within 6.5 years prior to self-reported diagnosis, however, a wider range of decreases in BMI occurred $\left(\beta_{\text {diabetes }}=1.294, P=0.0064 ; \beta_{\text {diabetes }} *\right.$ time $=$ $\left.0.150, P=0.0327 ; \beta_{\text {diabetes } * \text { time }}^{2}=-0.008, P=0.0065\right)$. The faster rate of increases in BMI followed by a greater decline was especially prominent in males and individuals with BMI $\geqq 24$.

CONCLUSIONS: An unintentional decrease in BMI in sharp contrast to the gradually rising BMI preceding that time may be an alarm for undiagnosed diabetes or a precursor to developing diabetes.

KEY WORDS: BMI; diabetes; older adults; trajectory; weight change.

Electronic supplementary material The online version of this article (doi:10.1007/s11606-016-3750-y) contains supplementary material, which is available to authorized users.

Received September 16, 2015

Revised April 21, 2016

Accepted May 9, 2016

Published online June 2, 2016

\author{
Abbreviations \\ BMI Body mass index \\ TLSA Taiwan longitudinal study on aging
}

J Gen Intern Med 31(10):1156-63

DOI: $10.1007 / \mathrm{s} 11606-016-3750-y$

(c) Society of General Internal Medicine 2016

\section{INTRODUCTION}

The prevalence of diabetes is increasing worldwide. ${ }^{1}$ Studies suggest that early detection and prompt treatment may be able to prevent or delay troubling complications and the related economic costs. ${ }^{2}$ Current screening procedures for diabetes include fasting plasma glucose, the 2-h plasma glucose after $75-\mathrm{g}$ oral glucose tolerance test, and hemoglobin A1c test ${ }^{3}$. However, people who do not habitually engage in regular medical checkups are unlikely to have these screenings. As a result, a large number of diabetes diagnoses are delayed, ${ }^{4}$ particularly in later adulthood. ${ }^{5}$ For this reason, helping adults living in the community to notice the early signs of diabetes and detect diabetes in its early stages has significant public health implications.

Recent studies analyzing trends in body weight before diagnoses of diabetes shed light on the possibility that weight changes may be an important variable for detecting the development of diabetes. ${ }^{6-8}$ These studies found there was a sudden drop in body weight 1 year prior to and after diabetes diagnosis, which was in sharp contrast to the gradually rising body weight preceding that time. However, existing studies only used regional data from participants who developed diabetes, and thus it was not possible to compare weight change patterns over time in a similar age group of adults who did not develop diabetes. Moreover, studies based on Western populations may not accurately represent patterns of body weight changes prior to diabetes diagnosis in Asians - a population in which BMI predicts outcomes differently ${ }^{9-11}$ and in which at similar BMI levels the prevalence of diabetes has been shown to be higher compared to Caucasians. ${ }^{12,13}$

This study examined long-term trajectories in BMI for individuals who did and did not develop diabetes in a retrospective cohort study, with participants aged $50+$ from the 
Taiwan Longitudinal Study on Aging. The objectives were: (1) to examine the change trajectories in BMI prior to selfreported diabetes diagnosis, including the timing and quantity, and (2) to see if these change trajectories differed from those seen among the group who did not develop diabetes. We also examined whether these patterns differed by sex and initial BMI.

\section{MATERIALS AND METHODS}

\section{Participants}

Data were drawn from a subset of three cohorts from the Taiwan Longitudinal Study on Aging (TLSA). We conducted a retrospective cohort study to determine whether BMI trajectories differed between those with and without diabetes in 2007. Full details regarding the recruitment procedures and characteristics of TLSA participants have been described previously. ${ }^{14}$ Briefly, the TLSA is a nationally representative longitudinal study of middle-aged and older adults living in Taiwan. Six waves of interviews were conducted in 1989, 1993, 1996, 1999, 2003, and 2007. Fielded in 1989, the first cohort comprised adults aged 60 and above at baseline (TLSA1989-2007, $n=4049$ ). The second and third cohorts were adults aged 50-66 in 1996 (TLSA1996-2007, $n=2462$ ) and adults aged 50-56 in 2003 (TLSA2003-2007, $n=1599$ ).

The present study combined all three cohorts: TLSA1989-2007, TLSA1996-2007, and TLSA20032007 (see online Appendix A, Table I). To ensure the validity of time of BMI, we excluded those who reported having diabetes in 2007 but were missing a diabetic status in $2003(n=19)$. In addition, the BMIs at the highest upper or lower $1 \%$ of the participants (i.e., BMI $\geq 60$ or $\leq 10 \mathrm{~kg} / \mathrm{m}^{2}$ ) were considered as outliers and thus excluded from further analysis $(n=64)$. Participants without diabetes diagnoses at baseline $(1989,1996$, or 2003) and who first reported diabetes diagnoses in 2007 were analyzed as individuals with the diagnoses of developing diabetes $(n=200$, a total of 760 personexaminations); those who reported the absence of diabetes at baseline and during the entire follow-up period became the reference group $(n=3576$, a total of 13,730 person-examinations).

\section{Measures}

Diabetes was our main explanatory variable; it was measured by the question "Have you been told by a physician that you have diabetes?" as asked in every interview wave. The primary response variable was BMI $\left(\mathrm{kg} / \mathrm{m}^{2}\right)$, which is a continuous measure calculated using self-reported height (meters) and weight (kilograms) at every examination. We chose BMI instead of weight because it is the measurement of body weight with various corrections for height. We performed a right adjustment on the point in time when diabetes diagnosis was self-reported. Specifically, time was coded to reflect the number of years before diabetes diagnosis in those who developed diabetes. For example, in participants without diabetes from 1989 until 2007, BMI in 2007 was coded as $\mathrm{BMI}_{\text {time }}=$ ${ }_{0}$, $\mathrm{BMI}$ in 2003 was coded as $\mathrm{BMI}_{\text {time }=-4}$, and so on. $\mathrm{BMI}$ can thus be traced up to 18 years before diabetes $\left(\mathrm{BMI}_{\text {time }}=-18\right.$ in 1989). The reference group was right adjusted using the same methods, and BMI in 2007 was coded as $\mathrm{BMI}_{\text {time }}=0$. This way the BMI trajectories before diabetes could be compared to approximately the same age reference group. A detailed description of the time axis for the developing diabetes group and diabetes free group, as well as the age comparability of the two groups, can be found in Table II in the online Appendix.

Covariates include sex, education, time-varying comorbidities, and lifestyle factors. Education was an ordered variable, defined as the number of years of education and drawn from the baseline survey. Comorbidities, including hypertension, heart disease, and stroke, indicated whether the participant reported that they had a physician diagnosis of the disease. Exercise was constructed from the reported frequency, including "often" ( $\geq 90 \mathrm{~min} /$ week), "sometimes" (1-89 $\mathrm{min} /$ week), or "scarcely" ( $0 \mathrm{~min} /$ week $)$. Alcohol consumption and smoking were categorized as current users versus nonusers by the participants' responses to the questions: "Do you drink in your daily life?" and "Do you smoke currently?" Because the diet control questionnaires were not included in every wave, we only used the items in the first interviews to represent the participants' diet control status for all waves. We placed participants who responded as having regulated their diets for health-related reasons into a "diet control" category.

\section{Data Analyses}

We used SAS 9.3 to perform all of the analyses. Bivariate analyses investigated the baseline characteristics of participants with and without developing diabetes, with chi-square tests for categorical variables and t-tests for continuous variables. Multilevel modeling (MLM) ${ }^{15}$ was used to depict the levels and rates of change in BMI up to 18 years before for the participants with a self-reported physician diagnosis of diabetes and for those without this diagnosis.

We determined the shape of BMI trajectories by comparing the model fits of the intercept only, linear, quadratic, and cubic growth curve models. By examining the changes in deviance scores $(-2 L L)$ from one model to another, we determined the model with the best fit. To examine potential moderating effects, we conducted sub-analyses by sex and baseline BMI. Based on the overweight criteria used in Taiwan, ${ }^{16}$ BMI $\geq 24$ was defined as "overweight" and BMI $<24$ was defined as "not overweight."

\section{RESULTS}

Data on BMI changes from 3776 older adults who were followed for up to 18 years $(14,490$ person-years $)$ were 
obtained in this study. The age range for the 3776 participants was $50-87$ years (mean $=58.41$ years, $S D=6.01)$ at baseline. Nearly half $(48.94 \%)$ of the participants were female. The mean BMI at baseline was $23.7(\mathrm{SD}=3.31)$. As Table 1 shows, we found no significant differences between those who developed diabetes $(n=200)$ and those who did not $(n=3576)$ in terms of age, sex, education, alcohol consumption, smoking, exercise, and heart disease at baseline. However, participants who developed diabetes were more likely to have hypertension $(34.00 \%$ vs. $17.11 \%, P<0.001)$ and stroke (2.50 \% vs. $1.03 \%, P=0.0545)$, a higher initial BMI (25.63 vs. $23.68, P<0.001$ ), and lower frequency of diet control $(47.50 \%$ vs. $55.73 \%, P=0.0228)$. All the variables on which we found differences, and those that may theoretically affect BMI, including age, sex, heart disease, and behavioral variables, were included as covariates in our subsequent MLM analyses.

The best shape of the BMI change trajectory before diabetes diagnosis was determined by comparing a series of nested models with the deviance test results. The results of the deviance test showed a substantial decrease in the deviance score (2log-likelihood, $-2 \mathrm{LL}$ ) when moving from the unconditional model to the linear model $\left(X_{(3)}^{2}=432.3, P<0.001\right)$. We found a similar result when moving from the linear model to the quadratic model $\left(X^{2}{ }_{(4)}=33.4, P<0.001\right)$. However, the cubic model did not converge in these samples, implying the quadratic model was the best fit for describing the BMI trajectory before physician-diagnosed diabetes.

Table 2 presents the MLM results of the BMI trajectory for adults who did or did not develop diabetes over the 18-year period. The results of the adjusted model were consistent with the raw model and showed that adults who did not develop diabetes had a rising but gradually slowing increase in BMI after age $50\left(\beta_{\text {time }}=0.08, P<0.001 ; \beta_{\text {time }}{ }^{2}=-0.002, P=\right.$ $0.0016)$. For adults who developed diabetes, the initial BMI (i.e., 18 years before diabetes) was significantly higher than that of the reference group $\left(\beta_{\text {diabetes }}=1.294, P=0.0064\right)$, and their BMI increased more rapidly $\left(\beta_{\text {diabetes*time }}=0.150, P=\right.$ $\left.0.0327 ; \beta_{\text {diabetes } * \text { time }}{ }^{2}=-0.008, P=0.0065\right)$ until approximately 6.5 years preceding the self-reported diabetes diagnosis (see note 1 under section B in the online Appendix for the calculation).

The results from the sub-analysis by sex show that the significantly higher initial BMI in adults developing diabetes was especially evident in female adults ( $\beta_{\text {diabetes }}=1.894, P=$ 0.020 in female; $\beta_{\text {diabetes }}=0.780, P=0.176$ in males). Females who developed diabetes had a $1.894 \mathrm{~kg} / \mathrm{m}^{2}$ higher initial BMI than the reference group. Further, male adults who developed diabetes had a significantly greater increase in BMI followed by a greater decline $\left(\beta_{\text {diabetes*time }}=0.163, P=\right.$ $\left.0.0561 ; \beta_{\text {diabetes }}^{*}{ }^{2}{ }^{2}=-0.008, P=0.0339\right)$ approximately 5.65 years before physician-diagnosed diabetes than females who developed diabetes (note 2 under section B in the online Appendix).

The results of the analysis that split the participants by their initial BMI into overweight (BMI $>=24 \mathrm{~kg} / \mathrm{m}^{2}$ ) and nonoverweight groups $\left(\mathrm{BMI}<24 \mathrm{~kg} / \mathrm{m}^{2}\right)$ indicated that within each group, those adults who developed diabetes did not have a significantly higher baseline BMI compared to their counterparts within the same weight category who did not develop

Table 1 Baseline Characteristics of Participants by Diabetes Development Status

\begin{tabular}{|c|c|c|c|c|}
\hline & $\begin{array}{l}\text { All } \\
(n=3776) \\
\% \text { or means } \pm \text { SD }\end{array}$ & $\begin{array}{l}\text { Developing diabetes group } \\
(n=200) \\
\% \text { or means } \pm \text { SD }\end{array}$ & $\begin{array}{l}\text { Diabetes-free group } \\
(n=3576) \\
\% \text { or means } \pm \text { SD }\end{array}$ & t-/chi-square \\
\hline Age & $58.41 \pm 6.01$ & $58.24 \pm 5.58$ & $58.42 \pm 6.04$ & -0.42 \\
\hline Female & 48.9 & 50.5 & 48.9 & 0.21 \\
\hline Education (years) & $6.64 \pm 4.64$ & $6.52 \pm 4.39$ & $6.64 \pm 4.65$ & -0.33 \\
\hline BMI $\left(\mathrm{kg} / \mathrm{m}^{2}\right)$ & $23.7 \pm 3.31$ & $25.63 \pm 3.63$ & $23.68 \pm 3.26$ & $7.40^{* * *}$ \\
\hline Current cigarette smoking & & & & 0.32 \\
\hline Yes & 27.2 & 25.5 & 27.3 & \\
\hline No & 72.8 & 74.5 & 72.7 & \\
\hline Daily alcohol consumption & & & & 0.09 \\
\hline Yes & 30.9 & 30.0 & 31.0 & \\
\hline No & 69.1 & 70.0 & 69.0 & \\
\hline Diet control & & & & $5.19^{*}$ \\
\hline Yes & 55.3 & 47.5 & 55.7 & \\
\hline No & 44.7 & 52.5 & 44.3 & \\
\hline Exercise & & & & 0.42 \\
\hline High & 43.6 & 42.0 & 43.7 & \\
\hline Middle & 16.0 & 17.5 & 15.9 & \\
\hline Low & 40.4 & 40.5 & 40.4 & \\
\hline Hypertension & & & & $36.58^{* * *}$ \\
\hline Yes & 18.0 & 34.0 & 17.1 & \\
\hline No & 82.0 & 66.0 & 82.9 & \\
\hline Heart disease & & & & 0.001 \\
\hline Yes & 9.9 & 10.0 & 9.9 & \\
\hline No & 90.1 & 90.0 & 90.1 & \\
\hline Stroke & & & & $3.70^{\dagger}$ \\
\hline Yes & 1.1 & 2.5 & 1.0 & \\
\hline No & 98.9 & 97.5 & 99.0 & \\
\hline
\end{tabular}

${ }^{* * *} p<0.001,{ }^{* *} p<0.01,{ }^{*} p<0.05,{ }^{\dagger} p<0.08$ 


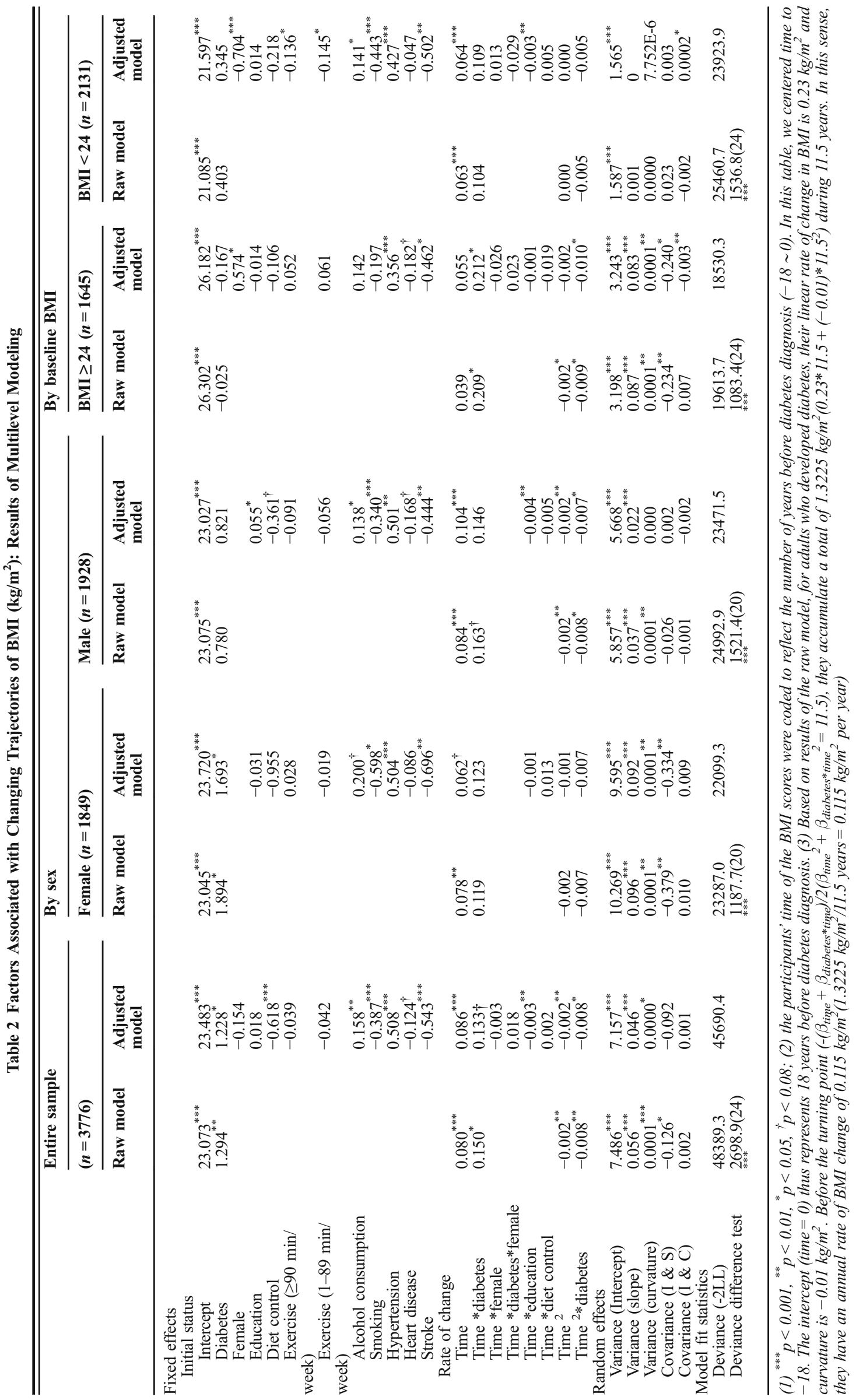


diabetes. In addition, the faster rate of BMI increase followed by an earlier decrease in BMI before diabetes was only present in overweight adults: In the overweight group that developed diabetes, the BMI trajectory had a clear upward trend that changed direction approximately 8.5 years preceding selfreported diabetes diagnosis (note 3 under section $B$ in the online Appendix).

In adults with an initial $\mathrm{BMI}<24$, their BMI trajectory pattern did not significantly differ by diabetes status $\left(\beta_{\text {diabetes*time }}=0.104, P=0.1642, \beta_{\text {diabetes*time }}{ }^{2}=-0.005\right.$, $P=0.127)$ - their BMI increased $\left(\beta_{\text {time }}=0.063, P<0.001\right.$; $\left.\beta_{\text {time }}{ }^{2}=-0.0004, P=0.4981\right)$ during the 18 -year follow-up in both the developing diabetes group and the reference group. Figure 1a illustrates the 18-year BMI trajectories before selfreported diabetes diagnosis and compares these with those of individuals who did not develop diabetes, while Fig. $1 \mathrm{~b}$ and $\mathrm{c}$ shows the trajectories by sex and baseline BMI, respectively.

Finally, based on the random effects, in the "female" and "overweight" groups the intercept and slope had a negative correlation (covariance $_{\mathrm{I}, \mathrm{S}}=-0.379, P=0.0018$, in the female group; covariance ${ }_{\mathrm{I}, \mathrm{S}}=-0.234, P=0.0086$, in the overweight group), indicating that a lower initial BMI may be associated with a more rapid increase in BMI from midlife to older adulthood in women or those who are overweight at baseline.

\section{DISCUSSION}

There is limited population-based evidence concerning the variations in weight prior to developing diabetes or at the time of diabetes diagnosis. By examining the BMI records of a nationally representative sample of adults aged 50 and above at baseline in Taiwan followed for 18 years, and by comparing those who did and did not develop diabetes during the followups, this study reveals possible patterns that could be used for early detection of diabetes. Independent of aging, timevarying behavioral and morbidity variables, a higher BMI, and a faster rate of weight gain were present more than 6.5 years before self-reported diabetes diagnosis. Within 6.5 years prior to self-reported diagnosis, however, a greater amount of weight loss occurred.

Long-term increases in body weight tend to lead to an increased risk of diabetes. ${ }^{6,17-25}$ However, not all research concludes that diabetes and a fall in body weight are closely related. Some studies find that weight loss can lead to a reduction in diabetes risk, ${ }^{20,21,24}$ while others find no connection. ${ }^{19,26}$ One possibile explanation for this inconsistency in the literature stems from the problem of measurement timing. Our study employed multilevel modeling to estimate annual changes in BMI. We plotted a long-term BMI growth curve that describes the relationship between BMI and the development of diabetes at different time points. For example, based on our estimated coefficients in Table 2 or the note beneath Fig. 1a, an individual with a height of $1.7 \mathrm{~m}$ at the mean age of 58.4 years old and without diabetes will gain $2.29 \mathrm{~kg}$ in
18 years (i.e., about $0.13 \mathrm{~kg}$ per year). In contrast, adults who are developing diabetes will gain a total of $3.82 \mathrm{~kg}$ during the 18- to 6.5-year period before diagnosis of diabetes (i.e., $0.33 \mathrm{~kg}$ per year) and then lose a total of $1.25 \mathrm{~kg}$ within 6.5 years preceding diabetes diagnosis (i.e., about $0.19 \mathrm{~kg}$ per year). Details of the calculations can be found in the online Appendix.

Looker and colleagues examined the BMI and weight of adult Pima Indians aged 15 and older living in the Gila River Community in Central Arizona. ${ }^{6}$ They reported that prior to receiving a diagnosis of diabetes (based on the 75-g oral glucose tolerance test), patients exhibited an average increase in BMI of $0.43-0.71 \mathrm{~kg} / \mathrm{m}^{2}$ annually and then lost weight after diagnosis. ${ }^{6}$ Although this study described patients' BMI changes up to 25 years before and after the diagnosis of diabetes, it did not compare the changes in BMI patterns with those from participants who did not develop diabetes, nor did it assess whether the weight gain patterns differed by weight groups or sex. In our study, individuals who developed diabetes showed increases in BMI of $0.115 \mathrm{~kg} / \mathrm{m}^{2}$ per year (linear rate of change is $0.23 \mathrm{~kg} / \mathrm{m}^{2}$; curvature is $-0.01 \mathrm{~kg} / \mathrm{m}^{2}$ ) before 6.5 years prior to self-reported physician diagnosis. This finding was even more pronounced in male participants, with a BMI increase of $0.123 \mathrm{~kg} / \mathrm{m}^{2}$ per year (the linear rate of change is $0.247 \mathrm{~kg} / \mathrm{m}^{2}$; curvature is $-0.01 \mathrm{~kg} / \mathrm{m}^{2}$ ) before 5.65 years prior to self-reported physician diagnosis. Our numbers are much lower than those in Looker et al.'s research on adult Pima Indians. One possible explanation for this discrepancy is that Looker's participants were younger than our middle-aged and older subjects, while another possibility is race/ethnicity. To standardize the rate of change, we divided the rate of increase by baseline BMI and found there was a 1.2-2.1\% annual BMI increase (BMI increase per year/initial $\mathrm{BMI}=0.43 \sim 0.71 / 34.6=1.2 \sim 2.1 \%$ ) in Looker's study compared to a $0.5 \%$ BMI increase in our work (BMI increase per year/initial BMI $=0.115 / 23.7=0.5 \%$ ).

Previous research suggests older adults tend to experience weight loss over time. ${ }^{27,28}$ Interestingly, our analyses reveal that those who developed diabetes exhibited weight loss at an earlier age, and the weight loss was at a more rapid rate: participants who developed diabetes lost a total of $0.423 \mathrm{~kg} / \mathrm{m}^{2}$ within the 6.5 years before their self-reported diagnosis. This finding supports and extends the results from Olivarius and colleagues. ${ }^{7,8}$ They recorded body weights prior to the diagnosis of diabetes and found there was a sudden drop in body weight 1 year prior to this diagnosis. We posit two explanations for the wider range in time with regard to the years when weight loss started to be observed before diabetes development. First, this may be linked to undiagnosed diabetes in these participants, as previous research identified that there is usually a delay in diabetes diagnosis of 4-7 years. ${ }^{4}$ Second, a reduction in BMI may be the result of other physiological changes during the pre-diabetes stage, ${ }^{29}$ such as the beta-cell 
a

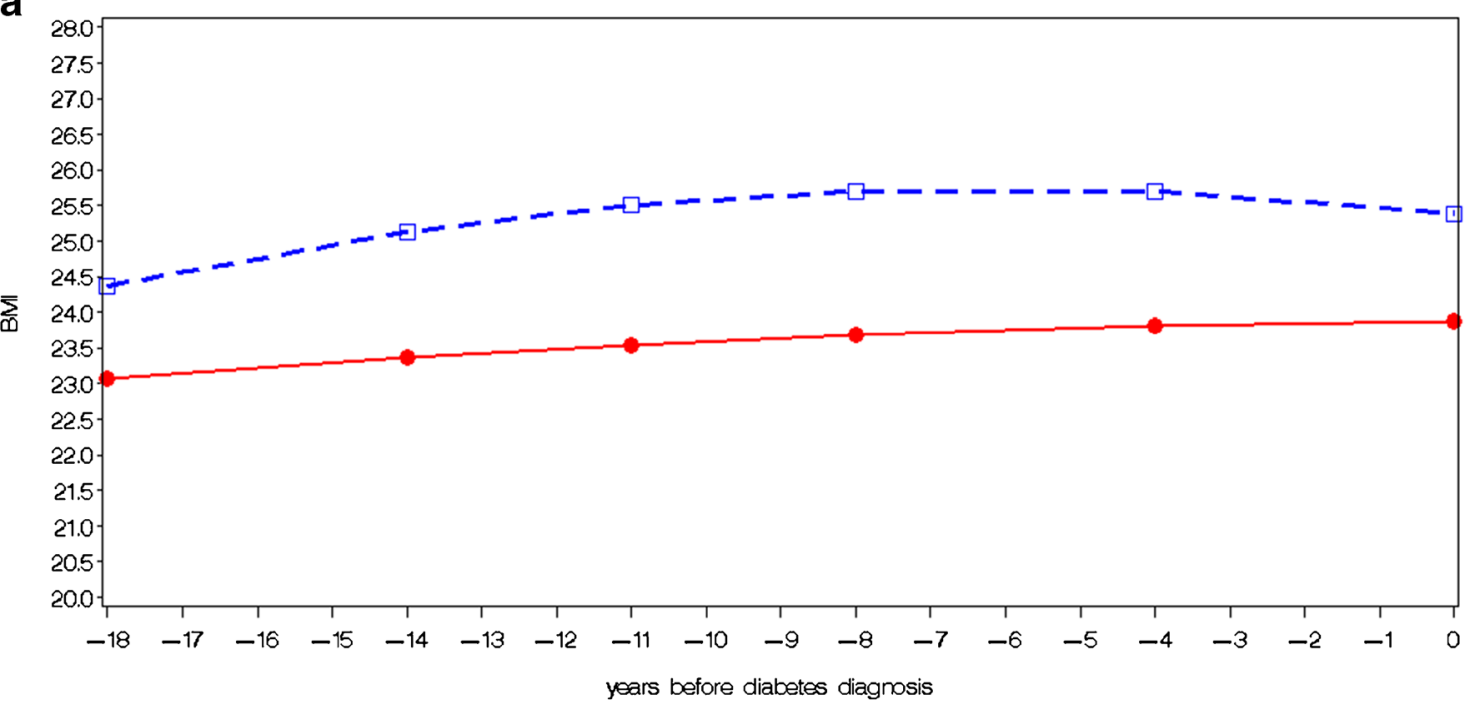

Entire Sample Developed Diabetes $\cdots$ Free from Diabetes

b
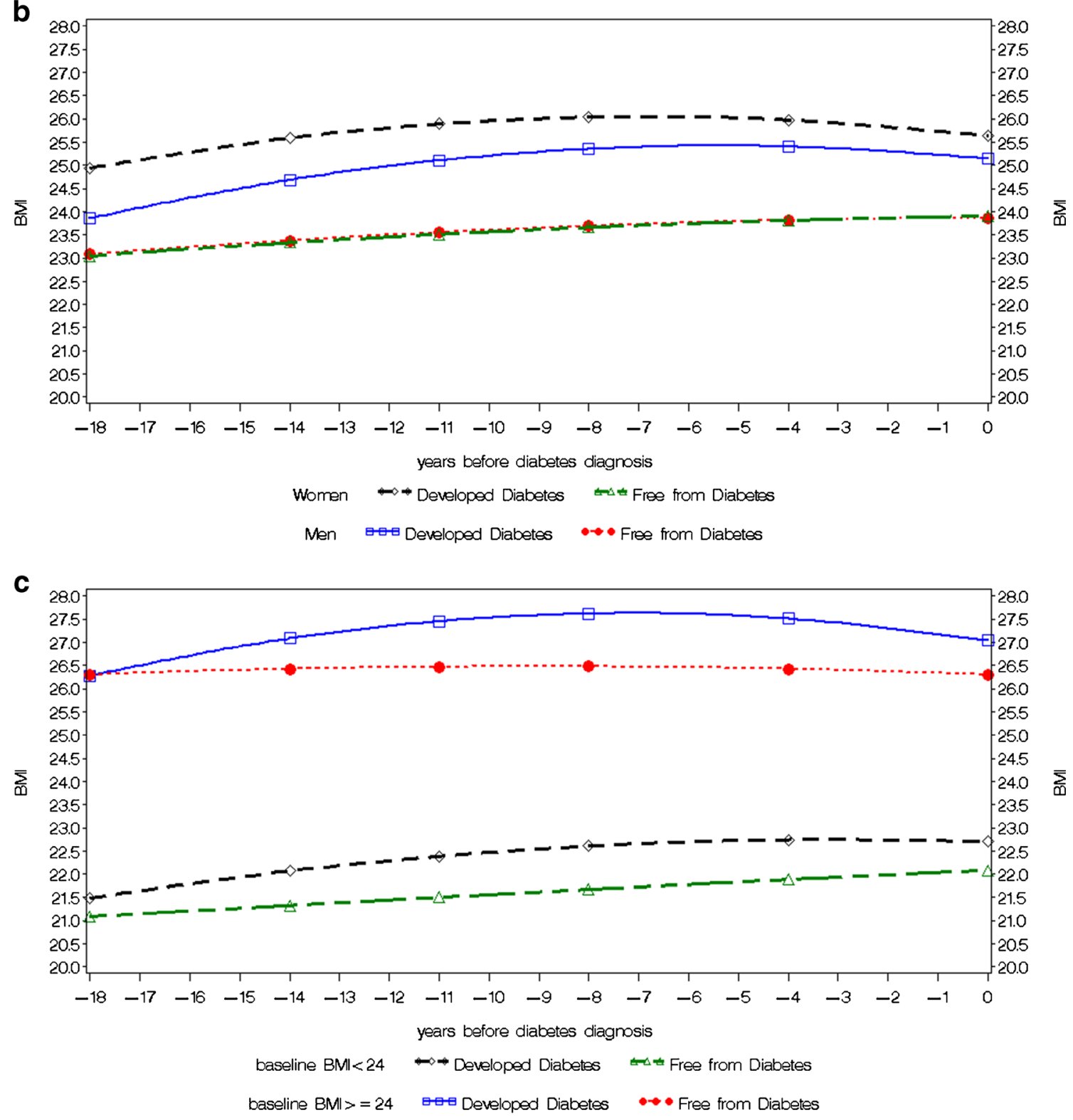
4 Fig. 1 a BMI trajectories before self-reported diabetes diagnosis in adults with and without developing diabetes. Note: For the diabetesfree group, $\mathrm{BMI}=\mathbf{2 3 . 0 7 3}+\mathbf{0 . 0 8 0} *$ time $-\mathbf{0 . 0 0 2} *$ time $^{2}$. For the developing diabetes group, $\mathrm{BMI}=(\mathbf{2 3 . 0 7 3}+\mathbf{1 . 2 9 4})+(\mathbf{0 . 0 8 0}+\mathbf{0 . 1 5 0})$ * time- $(0.002+0.008) *$ time $\left.^{2}\right)$. b BMI trajectories before selfreported diabetes diagnosis in males and females with and without developing diabetes. Note: For female + diabetes-free group, $\mathbf{B M I}=$ $23.045+0.078 *$ time. For male + diabetes-free group, $B M I=$ $23.075+0.084 *$ time $-0.002 *$ time $^{2}$. For female + developing diabetes group, $\mathrm{BMI}=(23.045+1.894)+0.078 *$ time. For male + developing diabetes group, $\mathrm{BMI}=\mathbf{2 3 . 0 7 5}+(\mathbf{0 . 0 8 4}+\mathbf{0 . 1 6 3}) *$ time $-(0.002+0.008) *$ time $^{2}$. c BMI trajectories before self-reported diabetes diagnosis in individuals with and without developing diabetes by initial body size. Note: For $\mathrm{BMI}<\mathbf{2 4}$ + diabetes-free group, BMI $=21.085+0.063 *$ time. For $B M I \geq 24+$ diabetes-free group, $\mathrm{BMI}=\mathbf{2 6 . 3 0 2}-\mathbf{0 . 0 0 2} *$ time $^{2}$. For $\mathrm{BMI}<24+$ developing diabetes group, $\mathrm{BMI}=\mathbf{2 1 . 0 8 5}+\mathbf{0 . 0 6 3}$ * time. For $\mathrm{BMI} \geq \mathbf{2 4}+$ developing diabetes group, BMI $=\mathbf{2 6 . 3 0 2}+0.209 *$ time $-(0.002+$ 0.009) * time $^{2}$

dysfunction and insulin resistance that prevent blood glucose utilization by cells and promote gluconeogenesis through muscle proteolysis. ${ }^{30}$

The differences in BMI and development of diabetes in Asian populations have received a significant growth in interest recently. A number of studies have thus discussed the BMI cutoff points needed to identify at-risk Asian-Americans for type 2 diabetes screening and pointed out the urgent need to increase early detection. ${ }^{13,31}$ Although these studies suggested BMI $\geqq 23$ as the most sensitive cutoff point for identifying Asian-Americans at elevated risk of diabetes, they also noted the tremendous heterogeneity among the various AsianAmerican subgroups and the complexity of applying an optimal BMI cutoff point of detecting diabetes development. By analyzing a nationally representative sample of older Taiwanese adults with and without development of diabetes over up to 18 years, our study adds value to this line of research. We found that the predictive values of being overweight, obese, or having an increased rate of weight gain associated with the development of type 2 diabetes may not be the same across individuals with different body size or sex. The overweight pattern associated with diabetes diagnosis is especially evident in females (females had an average of $1.894 \mathrm{~kg} / \mathrm{m}^{2}$ higher BMI than their female counterparts not developing diabetes); the faster rate of weight gain associated with diabetes diagnosis was especially prominent in males and individuals with baseline $\mathrm{BMI} \geqq 24$. In addition, among those whose BMI $\geq 24$ $\mathrm{kg} / \mathrm{m}^{2}$, their upward BMI trend that changed directions 8.5 years preceding self-reported diabetes diagnosis was longer than for the entire sample, which was 6.5 years. This suggests that underdiagnosed diabetes may be more prevalent in adults who are overweight or obese. Furthermore, we found that among those participants whose BMI $<24 \mathrm{~kg} / \mathrm{m}^{2}$ at baseline, neither the higher level nor the greater increases in BMI were associated with the development of diabetes, thus providing support for studies that indicate there are complex mechanisms at work in individuals who are normal weight or underweight but then develop diabetes. ${ }^{32,33}$ Whether weight fluctuations or other mechanisms are associated with the development of diabetes in adults who are underweight or normal weight is an issue that needs more research attention.

A major strength of the present study is its 18-year followup period based on a nationally representative sample of adults aged 50+ who did and did not develop diabetes. This allows us to investigate longitudinal changes in BMI in a heterogeneous group of older adults based on the same participants and provides information that can help raise awareness of the importance of early detection of diabetes for adults living in the community. In addition, unlike studies ${ }^{6,7,34}$ that did not have age-appropriate controls to enable the detection of BMI differences between those who did and did not develop diabetes at the same age, the present study matched the diabetes group with a comparable group of the same age to contrast the BMI trajectories over time. Furthermore, by controlling for BMI-related covariates over time, such as lifestyle and comorbidities, the present study was able to ascertain the impact of diabetes on the changes in BMI more precisely. Finally, this study was based on Taiwanese participants and thus adds value to the epidemiologic literature with regard to better understanding the BMI change history before self-reported diabetes diagnosis in an older Asian population aged 50 and above.

Some limitations exist for the current work and should be considered in the interpretation of its findings. One issue is related to the self-reported data on diabetes and the interview intervals of 3 to 4 years, with a right adjustment on the point in time when diabetes was self-reported. Although assessment of self-reported accuracy in this database indicates a high level of consistency with regard to the self-reported diabetes status and related biomarkers, ${ }^{35}$ patients who are not on a medication for diabetes may not recall that they have been told that they have diabetes. Thus, the weight loss in the 6.5 years before selfreported diabetes diagnosis may include the stage of underdiagnosed diabetes or adults who start behavioral interventions after being diagnosed with diabetes. The adjustment of time-varying behavioral covariates can alleviate the bias related to this to some extent, but there may still be some overestimation with regard to the years when weight loss started to be observed before diabetes development. Similarly, although BMI calculated from self-reported height and weight is highly correlated with measured height and weight, ${ }^{36}$ it has been shown that subjects tend to overestimate the former and underestimate the latter. ${ }^{36}$ The level of BMI in this study may thus be underestimated, and future studies using other measures of adiposity will help shed light on this line of research.

In conclusion, and with the aforementioned caveats, the present study supports the possibility of including changes in BMI as an indicator for future diabetes screening. Having easy-to-understand pre-diabetes indicators that patients can monitor easily, such as sudden weight loss, can empower patients and lead to improved communication between patients and physicians. Because our analyses controlled for time-varying behavioral and morbidity covariates, it highlights that a constant increase in BMI, with an average of 
about $0.5 \%$ increase per year, followed by an unintentional weight loss, may be an alarm for underdiagnosed diabetes or a precursor to developing diabetes. In addition, in the population examined in this study, those individuals who did not develop diabetes had an average BMI of around $23 \mathrm{~kg} / \mathrm{m}^{2}$. Those individuals who developed diabetes during the follow-up period had an average BMI of about $24 \mathrm{~kg} / \mathrm{m}^{2}$ for males and $25 \mathrm{~kg} / \mathrm{m}^{2}$ for females; for those individuals who were not overweight at baseline, neither the higher level nor the greater increase in BMI were associated with the development of diabetes. These findings support the view that a lower BMI cutoff point is appropriate for identifying Asians who should be screened for undiagnosed type 2 diabetes ${ }^{13,31}$ and that a BMI between 24 and $25 \mathrm{~kg} / \mathrm{m}^{2}$ may be the most appropriate point for identifying at-risk Taiwanese adults for type 2 diabetes screening.

Acknowledgments: We thank Prof. Chi-Pang Wen for his invaluable comments on the earlier version of this manuscript. This work was supported by grants from the National Science Council of Taiwan (NSC101-2314-B-006-001-). The TLSA is conducted and supported by the Taiwan National Institute of Family Planning (now the Bureau of Health Promotion) in collaboration with the TLSA investigators. The authors acknowledge the immense contributions that this support made in creating and maintaining this data set.

Corresponding Author: Ching-Ju Chiu, PhD; Institute of Gerontology, College of MedicineNational Cheng Kung University, No. 1, University Road 70101 , Tainan, Taiwan (e-mail: cjchiu@mail.ncku.edu.tw).

\section{Compliance with Ethical Standards:}

Conflict of Interest: The authors declare that they do not have a conflict of intere.

\section{REFERENCES}

1. Shaw JE, Sicree RA, Zimmet PZ. Global estimates of the prevalence of diabetes for 2010 and 2030. Diabetes Res Clin Pract. 2010;87(1):4-14.

2. Harris MI, Eastman RC. Early detection of undiagnosed diabetes mellitus: a US perspective. Diabetes Metab Res Rev. 2000;16(4):230-236.

3. American Diabetes Association. 2. Classification and diagnosis of diabetes. Diabetes Care. 2016;39 Suppl 1:S13-22.

4. Harris MI, Klein R, Welborn TA, Knuiman MW. Onset of NIDDM occurs at least 4-7 yr before clinical diagnosis. Diabetes Care. 1992;15(7):815819.

5. Rathmann W, Haastert B. Icks AA, et al. High prevalence of undiagnosed diabetes mellitus in Southern Germany: target populations for efficient screening. The KORA survey 2000. Diabetologia. 2003;46(2):182-189.

6. Looker HC, Knowler WC, Hanson RL. Changes in BMI and weight before and after the development of type 2 diabetes. Diabetes Care. 2001;24(11):1917-1922.

7. de Fine Olivarius N, Richelsen B, Siersma V, Andreasen AH, BeckNielsen H. Weight history of patients with newly diagnosed type 2 diabetes. Diabet Med. 2008;25(8):933-941.

8. de Fine ON, Siersma VD, Koster-Rasmussen R, Heitmann BL, Waldorff FB. Weight changes following the diagnosis of type 2 diabetes: the impact of recent and past weight history before diagnosis. results from the Danish Diabetes Care in General Practice (DCGP) study. PLoS One. 2015;10(4):e0122219.

9. Huxley R, James W, Barzi F, et al. Ethnic comparisons of the crosssectional relationships between measures of body size with diabetes and hypertension. Obes Rev. 2008;9(s1):53-61.

10. Jee SH, Sull JW, Park J, et al. Body-mass index and mortality in Korean men and women. N Engl J Med. 2006;355(8):779-787.
11. Gu D, He J, Duan X, et al. Body weight and mortality among men and women in China. JAMA. 2006;295(7):776-783.

12. King GL, McNeely MJ, Thorpe LE, et al. Understanding and addressing unique needs of diabetes in Asian Americans, native Hawaiians, and Pacific Islanders. Diabetes Care. 2012;35(5):1181-1188.

13. Hsu WC, Araneta MR, Kanaya AM, Chiang JL, Fujimoto W. BMI cut points to identify at-risk Asian Americans for type 2 diabetes screening. Diabetes Care. 2015;38(1):150-158.

14. Chiu C-J, Wray LA, Ofstedal MB. Diabetes-related change in physical disability from midlife to older adulthood: Evidence from 1996-2003 Survey of Health and Living Status of the Elderly in Taiwan. Diabetes Res Clin Pract. 2011;91(3):413-423.

15. Raudenbush Sw, Bryk AS. Hierarchical linear models: applications and data analysis methods. Sage. 2002. 1.

16. Chu NF. Prevalence of obesity in Taiwan. Obes Rev. 2005;6(4):271-274.

17. Koh-Banerjee P, Wang Y, Hu FB, Spiegelman D, Willett WC, Rimm EB. Changes in body weight and body fat distribution as risk factors for clinical diabetes in US men. Am J Epidemiol. 2004;159(12):1150-1159.

18. Power C, Thomas C. Changes in BMI, duration of overweight and obesity, and glucose metabolism: 45 years of follow-up of a birth cohort. Diabetes Care. 2011;34(9):1986-1991.

19. Nanri A, Mizoue T, Takahashi Y, et al. Association of weight change in different periods of adulthood with risk of type 2 diabetes in Japanese men and women: the Japan Public Health Center-Based Prospective Study. J Epidemiol Community Health. 2011;65(12):1104-1110.

20. Wannamethee SG, Shaper AG, Walker M. Overweight and obesity and weight change in middle aged men: impact on cardiovascular disease and diabetes. J Epidemiol Community Health. 2005;59(2):134-139.

21. Resnick HE, Valsania $\mathbf{P}$, Halter JB, Lin $\mathbf{X}$. Relation of weight gain and weight loss on subsequent diabetes risk in overweight adults. J Epidemiol Community Health. 2000;54(8):596-602.

22. Hwang $\mathbf{L}-\mathbf{C}$, Chen $\mathbf{C}-\mathbf{J}$, Lin BJ. Obesity and changes in body weight related to 10-year diabetes incidence in women in Taiwan (1993-2003). Asia Pac J Clin Nutr. 2007;16(4):677-682.

23. Biggs ML, Mukamal KJ, Luchsinger JA, et al. Association between adiposity in midlife and older age and risk of diabetes in older adults. JAMA. 2010;303(24):2504-2512.

24. Colditz GA, Willett WC, Rotnitzky A, Manson JE. Weight gain as a risk factor for clinical diabetes mellitus in women. Ann Intern Med. 1995; 122(7):481-486.

25. Schienkiewitz A, Schulze MB, Hoffmann K, Kroke A, Boeing H. Body mass index history and risk of type 2 diabetes: results from the European Prospective Investigation into Cancer and Nutrition (EPIC)-Potsdam Study. Am J Clin Nutr. 2006;84(2):427-433.

26. Oguma Y, Sesso HD, Paffenbarger RS, Lee IM. Weight change and risk of developing type 2 diabetes. Obes Res. 2005;13(5):945-951.

27. Gazewood JD, Mehr DR. Diagnosis and management of weight loss in the elderly. J Fam Pract. 1998;47(1):19-25.

28. Williamson DF. Descriptive epidemiology of body weight and weight change in US adults. Ann Intern Med. 1993;119(7_Part_2):646-649.

29. Tabák AG, Jokela M, Akbaraly TN, Brunner EJ, Kivimäki M, Witte DR. Trajectories of glycaemia, insulin sensitivity, and insulin secretion before diagnosis of type 2 diabetes: an analysis from the Whitehall II study. Lancet. 2009;373(9682):2215-2221.

30. Wang XH, Mitch WE. Muscle wasting from kidney failure-a model for catabolic conditions. Int J Biochem Cell Biol. 2013;45(10):2230-2238.

31. Araneta MR, Kanaya AM, Hsu WC, et al. Optimum BMI cut points to screen asian americans for type 2 diabetes. Diabetes Care. 2015;38(5):814820.

32. Chiu C-J, Wray LA, Lu F-H, Beverly EA. BMI change patterns and disability development of middle-aged adults with diabetes: a dual trajectory modeling approach. J Gen Intern Med. 2013;28(9):1150-1156.

33. Sairenchi T, Iso H, Irie F, Fukasawa N, Ota H, Muto T. Underweight as a predictor of diabetes in older adults a large cohort study. Diabetes Care. 2008;31(3):583-584.

34. Vistisen D, Witte DR, Tabák AG, et al. Patterns of obesity development before the diagnosis of type 2 diabetes: the Whitehall II cohort study. PLoS Med. 2014;11(2):e1001602.

35. Goldman N, Lin I-F, Weinstein M, Lin Y-H. Evaluating the quality of selfreports of hypertension and diabetes. J Clin Epidemiol. 2003;56(2):148154.

36. McAdams MA, Dam RM, Hu FB. Comparison of self-reported and measured BMI as correlates of disease markers in US adults. Obesity. 2007;15(1):188. 